\title{
What is a disease? What is the disease clinical benign prostatic hyperplasia (BPH)?
}

\author{
Keong Tatt Foo ${ }^{1}$
}

Received: 30 November 2018 / Accepted: 18 February 2019 / Published online: 25 February 2019

(c) The Author(s) 2019

\begin{abstract}
Introduction Though clinical benign prostatic hyperplasia (BPH) is a common disease worldwide, there is still much confusion in the literature and the many clinical guidelines as to its definition. Often the disease is associated with lower urinary tract symptoms (LUTS) and managed according to only symptoms. This leads to undertreatment in some patients with severe bladder outlet obstruction (BOO) with no symptoms, and overtreatment in patients with LUTS but no clinical BPH. Definition of a Disease Fundamentally, a disease can be defined as an abnormal structure or function or a condition which may cause harm to the organism.

Definition of clinical BPH Thus, clinical BPH can be defined as prostate adenoma/adenomata, causing a varying degree of BOO, which may eventually cause harm to the patients. With this definition, we are then able to differentiate the disease clinical BPH from the many other less common causes of LUTS, and then treat it according to its severity.

Diagnosing clinical BPH Clinical BPH can be diagnosed with non-invasive ultrasound in the clinic, grading it according to the shape (intravesical prostatic protrusion) and size of the prostate.

Clinical Significance Treatment can then be planned according to the disease severity using our staging system that classifies severity according to the presence or absence of significant obstruction and bothersomeness of symptoms.

Conclusion This would lead to better individualised and cost-effective management of the disease clinical BPH.
\end{abstract}

Keywords Benign prostatic hyperplasia $\cdot$ Definition $\cdot$ Grading $\cdot$ Staging $\cdot$ Transabdominal ultrasound $\cdot$ Individualised care

\section{Introduction}

The term benign prostatic hyperplasia (BPH) has been reserved for histological diagnosis, and the term benign prostatic enlargement (BPE) is used to describe the disease as seen in the clinic [1]. However, small prostates can also cause obstruction and symptoms [2]. And more recently, this common disease has been termed as benign prostatic obstruction (BPO) where the cause of bladder outlet obstruction $(\mathrm{BOO})$ is thought to be due to the prostate [3]. But is $\mathrm{BPO}$ a disease?

Keong Tatt Foo

foo.keong.tatt@singhealth.com.sg

1 Department of Urology, Singapore General Hospital, Academia Level 5, 20 College Road, Singapore 169856, Singapore

\section{Definition of a disease}

A disease can be defined as an abnormal homeostasis in structure or function or a condition that may result in harm to the organism. High blood sugar per se is not diabetes mellitus. It is the result and not the cause of the disease. Rather, the cause is abnormal insulin metabolism of the islet cells. Similarly, BPO is the consequence and not the cause of clinical BPH; the cause is prostate adenoma/adenomata [4].

\section{Defining clinical BPH}

Thus, clinical BPH can be defined as prostate adenoma/ adenomata causing BOO that may cause harm to the bladder and eventually the kidneys. Our study has shown that obstruction depends more on the site than size of the adenoma [5]. A small adenoma at the bladder neck forming the middle lobe can cause significant obstruction to the bladder due to distortion of the funnelling effect of the normal 
bladder neck. On the other hand, an adenoma forming the lateral lobes can grow to a considerable size before causing obstruction by compressing the prostatic urethra. In flow dynamics, compression is less obstructive than distortion $[4,6]$.

Due to the slow progression of the disease, patients may get used to the symptoms and not have any complaints. They may not have lower urinary tract symptoms (LUTS) and present instead with sudden acute retention of urine or less commonly be found to have chronic retention with renal failure. Some may present with painless gross haematuria, or urinary infection. With the introduction of PSA in health screening, many patients may present with raised PSA.

Therefore, clinical BPH can be defined as prostate adenoma/adenomata causing a varying degree of BOO, with or without symptoms.

\section{Diagnosing clinical BPH}

With the above definition, clinical BPH can nowadays be diagnosed with non-invasive ultrasound by measuring intravesical prostatic protrusion (IPP) in the clinic. IPP is measured in the sagittal view of a comfortably full bladder (about $200 \mathrm{ml}$ ) and it is the distance in $\mathrm{mm}$ from the innermost protrusion of the prostate perpendicularly down to the base at the circumference of the bladder [7]. It has a $100 \%$ positive predictive value and $100 \%$ specificity for diagnosing prostate adenoma [5]. Together with uroflowmetry, prostate adenoma can be ruled out as a cause of LUTS if there is no IPP and the flow rate is good [8]. This is useful in the differential diagnosis of male LUTS.

Prostate-specific antigen (PSA) is also important in the differential diagnosis. In patients with no clinical BPH, no prostatitis and no prostate cancer, PSA is generally less than $1 \mu \mathrm{g} / \mathrm{l}[6]$. This is seen in patients after transurethral enucleation of the prostate adenoma/adenomata) [9]. PSA is related to the size of the adenoma and in early studies investigating normal PSA levels, many of the subjects might have prostate adenoma of varying sizes with no LUTS symptoms, leading to PSA less than $4 \mu \mathrm{g} / \mathrm{l}$ being accepted as normal and the misconception that PSA increases with age. In contrast, we have observed in our clinic that if a patient has no clinical BPH (adenoma), no prostatitis and no cancer, the PSA level remains the same over the years [6].

\section{Clinical relevance}

With the new definition of clinical BPH, further assessment is done to determine whether there is possible harm to the bladder and kidneys. When the voiding function is affected, persistent post-void residual urine of more than
$100 \mathrm{ml}$ is observed, whereas when the storage function is affected, the maximum voided volume is low, less than $100 \mathrm{ml}$ [10]. Both parameters can be easily measured in the clinic. Obstruction is considered as significant when the above dysfunctions are present. The severity of the symptoms can be assessed with the International Prostate Symptoms Score (IPSS) and the quality of life (QoL) index. The QoL index is more important than IPSS and a QoL score of 3 and above is considered as bothersome [11].

Thus the severity of clinical BPH can be staged accordingly:

Stage I: no significant obstruction and no bothersome symptoms;

Stage II: no significant obstruction but has bothersome symptoms;

Stage III: significant obstruction irrespective of symptoms; and

Stage IV: complications of clinical BPH such as retention of urine, recurrent haematuria, urinary tract infection, and bladder stones.

Prostate adenoma/adenomata can be graded according to IPP (grade 1: $\leq 5 \mathrm{~mm}$, grade 2: $>5 \mathrm{~mm}-10 \mathrm{~mm}$, and grade $3:>10 \mathrm{~mm})$ and prostate volume $(a: \leq 20 \mathrm{~g}, b:>20-40 \mathrm{~g}$, and $c:>40 \mathrm{~g}$ ). Grade 1a prostates are least obstructive and grade 3a prostates most obstructive, and the disease can be managed accordingly [12]. Grade 3 a prostates would generally be better treated surgically, as they are small and easily removed by transurethral resection whereas grade 1c prostates would be more suitable for medical treatment with fivealpha reductase inhibitors.

About $60 \%$ of the patients with clinical BPH have lowgrade and low-stage disease and they can be watched with advice on fluid adjustment, diet, and exercise while those with higher stage and higher grade disease would need more aggressive medical or surgical treatment options [13, 14].

For patients with discordant grade and stage, urodynamic studies may still be necessary to assess obstruction and underactive detrusor dysfunctions, especially if more invasive treatment is contemplated. This discordance occurs in the minority, as shown in a study of 408 patients [13]. Of 44 patients in stage III, 37 patients had grade 3 IPP; only seven patients (16\%) had an IPP grade less than 3 .

Overactive bladder $(\mathrm{OAB})$ may be suspected from storage symptoms of urgency and frequency. If a patient has a high grade IPP of 3 (more than $10 \mathrm{~mm}$ ) and low maximum voided volume (less than $100 \mathrm{ml}$ ), he should be offered treatment with options for surgery or five-alpha reductase inhibitors if the prostate is more than $30 \mathrm{~g}$. Anticholinergics may cause more harm to patients, increasing post-void residual urine or even leading to retention of urine [10].

In another study of 114 patients with pressure flow study done, IPP was the best predictor for $\mathrm{BOO}$, with the positive 
predictive value at $72 \%$ compared to PV and PSA at $65 \%$ and $68 \%$, respectively [15].

\section{Conclusion}

The main objective of this review is to elucidate the definition of the disease clinical BPH. The focus is not on LUTS/BPH, which has been reviewed previously by our group $[16,17]$ and the other authors [18].

In medical school, we are taught to treat the disease, and not symptoms. Yet, currently many international guidelines still manage this common condition of male LUTS/BPH according to symptoms, using the International Prostate symptoms score (IPSS). Likewise, pharmaceutical and medical device companies use IPSS to gauge patients' response to treatment.

With the definition that clinical BPH is essentially a benign neoplasm (adenoma/adenomata), which may progress and eventually cause harm to the patient due to obstruction, BPO would be more important than the symptoms (IPSS). Therefore, obstruction should be given more weight in decision making in treating the disease according to severity. This can be done by the staging classification based on the presence or absence of significant obstruction and bothersomeness of symptoms. With a proper definition, the disease can be diagnosed and classified accordingly and treated appropriately.

Apart from history and physical examination, diagnosis in the clinic can be done more precisely with non-invasive ultrasound and the disease classified according to the grade of IPP to predict its course, as in malignant neoplasm. This would help to individualise management of the disease in the clinic, while also taking into account the patient's age, comorbidity and preferences for cost-effective personalised care.

Acknowledgements The author would like to thank nephrologist Prof Keng Thye Woo for his advice; Ms Mei Ying Ng and Dr Hong Hong Huang for their editorial assistance; and Lee Foundation, Goh Foundation and the National Medical Research Council, Singapore for their funding to support the BPH database in the Department of Urology, Singapore General Hospital.

Author contributions Keong Tatt Foo: manuscript writing.

\section{Compliance with ethical standards}

Conflict of interest The author declares that he has no conflict of interest.

Research involving human participants and/or animals Not applicable as this a review article.

Informed consent Not applicable as this is a review article.
Open Access This article is distributed under the terms of the Creative Commons Attribution 4.0 International License (http://creativeco mmons.org/licenses/by/4.0/), which permits unrestricted use, distribution, and reproduction in any medium, provided you give appropriate credit to the original author(s) and the source, provide a link to the Creative Commons license, and indicate if changes were made.

\section{References}

1. Abrams P, Cardozo L, Fall M, Griffiths D, Rosier P, Ulmsten U, Van Kerrebroeck P, Victor A, Wein A, Standardisation Sub-Committee of the International Continence Society (2003) The standardisation of terminology in lower urinary tract function: report from the standardisation sub-committee of the International Continence Society. Urology 61(1):37-49

2. Hirayama A, Samma S, Fujimoto K, Yamaguchi A, Akiyama T, Fukui Y (2002) Comparison of parameters to determine the cause of urinary disturbance in men with prostate volume less than 20 milliliters. Int J Urol 9(10):554-559

3. Gratzke C, Bachmann A, Descazeaud A, Drake MJ, Madersbacher S, Mamoulakis C, Oelke M, Tikkinen KAO, Gravas S (2015) EAU guidelines on the assessment of non-neurogenic male lower urinary tract symptoms including benign prostatic obstruction. Eur Urol 67(6):1099-1109

4. Foo KT (2017) Pathophysiology of clinical benign prostatic hyperplasia. Asian J Urol 4(3):152-157

5. Luo GC, Foo KT, Kuo T, Tan G (2013) Diagnosis of prostate adenoma and the relationship between the site of prostate adenoma and bladder outlet obstruction. Singap Med J 54(9):482-486

6. Foo KT (2016) Solving the benign prostatic hyperplasia puzzle. Asian J Urol 3(1):6-9

7. Tan YH, Foo KT (2001) Predicting the outcome of trial voiding following acute urinary retention using transabdominal ultrasound. J Urol 165(6):269

8. Foo KT, Lee LS (2010) Re: intravesical prostatic protrusion (IPP) and uroflowmetry in the management of benign prostatic enlargement (BPE). Int J Urol 17(6):589

9. Zhang KY, Xing JC, Chen BS, Liu CX, Lau HW, Sim HG, Foo KT (2011) Bipolar plasmakinetic transurethral resection of the prostate vs. transurethral enucleation and resection of the prostate: pre- and postoperative comparisons of parameters used in assessing benign prostatic enlargement. Singap Med J 52(10):747-687514

10. Singapore Urological Association Male Lower Urinary Tract Symptoms/Benign Prostatic Hyperplasia Guidelines Committee 2015 (2017) Singapore urological association clinical guidelines for male lower urinary tract symptoms/benign prostatic hyperplasia. Singapore Med J 58(8):473-480

11. Foo KT (1995) Current assessment and proposed staging of patients with benign prostatic hyperplasia. Ann Acad Med Singap 24(4):648-651

12. Wang D, Huang H, Law YM, Foo KT (2015) Relationships between prostatic volume and intravesical prostatic protrusion on transabdominal ultrasound and benign prostatic obstruction in patients with lower urinary tract symptoms. Ann Acad Med Singap 44(2):60-65

13. Wang D, Foo KT (2010) Staging of benign prostate hyperplasia is helpful in patients with lower urinary tract symptoms suggestive of benign prostate hyperplasia. Ann Acad Med Singap 39(10):798-802

14. Foo KT (2010) Decision making in the management of benign prostatic enlargement and the role of transabdominal ultrasound. Int $\mathbf{J}$ Urol 17(12):974-979

15. Lim KB, Ho H, Foo KT, Wong MY, Fook-Chong S (2006) Comparison of intravesical prostatic protrusion, prostate volume and serum prostatic-specific antigen in the evaluation of bladder outlet obstruction. Int J Urol 13(12):1509-1513 
16. Kuo TL, Teo JS, Foo KT (2016) The role of intravesical prostatic protrusion in the evaluation and treatment of bladder outlet obstruction (BOO). Neurourol Urodyn 35(4):535-537

17. Mangat R, Ho HSS, Kuo TLC (2018) Non-invasive evaluation of lower urinary tract symptoms (LUTS) in men. Asian J Urol 5(1):42-47

18. Bright E, Oelke M, Tubaro A, Abrams P (2010) Ultrasound estimated bladder weight and measurement of bladder wall thickness - useful noninvasive methods for assessing the lower urinary tract? J Urol 184(5):1847-1854

Publisher's Note Springer Nature remains neutral with regard to jurisdictional claims in published maps and institutional affiliations. 